\title{
APLICAÇÃO DA METODOLOGIA DO GRAU DE DETERIORAÇÃO DA ESTRUTURA (GDE / UNB) EM VIADUTOS DA CIDADE DE MANAUS: ESTUDO DE CASO
}

\author{
PANTOJA, DRISANA \\ Engenheira Civil \\ Universidade do Estado do Amazonas \\ Amazonas, Brasil \\ ddsp.eng@uea.edu.br
}

\author{
PINHEIRO, SAMANTHA \\ Professora \\ Universidade do Estado do Amazonas \\ Amazonas, Brasil \\ spinheiro@uea.edu.br
}

\section{RESUMO}

As obras de arte especiais são elementos estruturais destinados a vencer obstáculos, sejam estes naturais ou artificiais, para transposição do tráfego viário. Normalmente, localizados em áreas cujo tráfegos são intensos, as pontes e viadutos, em muitos casos não recebem a manutenção adequada, propiciando assim, o surgimento de manifestações patológicas. Neste contexto, este trabalho propôs a aplicação da metodologia do Grau de deterioração da estrutura (GDE/UnB), para avaliar viadutos da zona Centro-Sul da cidade de Manaus. Esta metodologia está baseada em inspeções visuais, para identificação de patologias causadas pelo tráfego, pelas intempéries e pela falta de manutenção, e em cálculos que permitam a quantificação do nível de deterioração que a estrutura apresenta. De acordo com as inspeções visuais tornouse possível a classificação de cada viaduto, o viaduto Miguel Arraes, sendo classificado com uma deterioração de nível médio. O complexo viário Plínio Ramos, contempla dois viadutos que atingiram deterioração de nível médio, o viaduto Duca Brito e Dom Jackson Damasceno possuem deterioração nível médio O viaduto Ayrton Senna apresentou o maior grau de deterioração, em nível sofrível. Ao comparar os valores com o estado atual dos viadutos observa-se que a metodologia é eficiente para aplicação in loco por meio de analises visuais pois retrata a realidade, vale ressaltar que mesmo as estruturas que alcançaram como resultado grau de deterioração médio há itens com deterioração mais elevados. Palavras-chave: Patologia. Viaduto. Deterioração.

\section{ABSTRACT}

Special works of art are structural elements intended to overcome obstacles, whether natural or artificial, for the transposition of road traffic. Usually located in areas where traffic is intense, bridges and viaducts, in many cases do not receive adequate maintenance, thus leading to the appearance of pathological manifestations. In this context, this work proposed the application of the Structure deterioration degree (GDE / UnB) methodology, to evaluate viaducts of the Center-South zone of the city of Manaus. This methodology is based on visual inspections, to identify pathologies caused by traffic, weather and lack of maintenance, and on calculations that allow the quantification of the level of deterioration that the structure presents. According to visual inspections it became possible to classify each viaduct, the Miguel Arraes viaduct, being classified with a medium level deterioration. The Plínio Ramos road complex has two viaducts that have reached medium level deterioration, the Duca Brito and Dom Jackson Damasceno viaduct have medium level deterioration. When comparing the values with the current state of the viaducts, it is observed that the methodology is efficient for on-site application through visual analysis because it portrays the reality, it is noteworthy that even the structures that achieved as a result of average deterioration level have items with deterioration. higher.

Keywords: Pathology. Viaduct. Deterioration.

\section{INTRODUÇÃO}

As Obras de Artes Especiais (OAE), como são conhecidas as pontes e viadutos, são elementos estruturais que permitem o deslocamento de tráfegos viários e, consequentemente, de pessoas sobre barreiras naturais como rios, braços de mar e regiões de relevo acentuado (MEDEIROS, 2015). Frequentemente situadas em áreas cujos tráfegos são intensos, esses importantes acervos técnicos são indispensáveis para a vida cotidiana de suas populações pois, definem os principais escoamentos das vias, articulando cruzamentos de grandes avenidas, encurtando caminhos e compensando acidentes geográficos (BASTOS e MIRANDA, 2017).

Todavia, apesar de sua grande importância econômica e social, a falta de manutenção propicia o surgimento de manifestações patológicas, que reduzem a vida útil das construções e comprometem a segurança de milhares de pessoas que utilizam desses meios diariamente (BARBOSA et al. 2017).

A ausência de manutenção das OAEs brasileiras acarreta em diferentes tipos de patologias como fissuras, manchas por eflorescência, exposição das armaduras, desagregação de concretos que, ao longo do tempo, comprometem a segurança 
estrutural, funcionalidade e durabilidade da estrutura. Além disso, o tipo e a intensidade da patologia sofrem influência de ações físicas e químicas diversas do ambiente na qual estão inseridas as OAEs (ALBUQUERQUE e OTOCH, 2015; LACERDA,2016).

A metodologia do grau de deterioração da estrutura (GDE/UnB) consiste na subdivisão da obra de arte especial em seus elementos componentes, como pilares, vigas, juntas de dilatação, para análise isolada. Esses elementos formam um grupo denominado família de elemento para análise em grupos e em seguida é executada a analise global da estrutura. Neste contexto, o presente trabalho se propôs a aplicar a metodologia desenvolvida por Castro (1994) e adaptada por Euqueres (2011), que determina o grau de deterioração que essa estrutura pode alcançar. Assim foi determinado o grau de deterioração em função das manifestações patológicas observadas em cinco viadutos da zona Centro-Sul da cidade de Manaus.

\section{METODOLOGIA}

\subsection{Metodologia GDE/UnB}

Neste trabalho adotou-se a metodologia desenvolvida por Euqueres (2011). Nesta metodologia as obras de artes especiais são divididas em seus elementos constituintes para analise isolada. Esses elementos formam grupos denominados família de elemento para analise em grupos, em seguida é executada a analise global da estrutura, para determinação do seu grau de deterioração. Abaixo são apresentados os fatores utilizados na metodologia

\subsubsection{Fator de ponderação $\left(\mathbf{F}_{\mathbf{p}}\right)$}

Este fator considera o provável grau de comprometimento estrutural ou de desempenho causado por uma manifestação patológica sobre um elemento, ou seja, a relevância estrutural da manifestação apresentada para um bom desempenho do elemento estrutural analisado (EUQUERES, 2011). Para cada dano especifico, e em função do elemento que apresenta o dano é pré-estabelecido por família uma nota dentro de uma escala de 1 a 5 . No item fissuras, o vistoriador estipula qual a importância da fissura apresentada na inspeção, podendo apresentar variações em sua escala de 2 a 5.

\subsubsection{Fator de intensidade do dano $\left(F_{i}\right)$}

O Fator de intensidade do dano (Fi) é atribuído no momento da vistoria, considerando a intensidade de cada manifestação patológica encontrada em cada elemento, o valor atribuído se encontra em uma escala de 0 a 4 , onde 0 é o valor atribuído ao elemento que não possui nenhum tipo de dano daquela natureza e 4 valor atribuído ao elemento que apresenta aquela lesão em estado péssimo (crítico), para o auxílio dessas atribuições utiliza-se a Tabela 1 (MEDEIROS, 2015).

Tabela 1 - Classificação dos danos e fatores de intensidade.

\begin{tabular}{c|c|c}
\hline \multirow{2}{*}{ Tipos de danos } & $\begin{array}{c}\text { Fator de } \\
\text { intensidade }\end{array}$ & Tipos de manifestação \\
\cline { 2 - 3 } & 0 & Sem lesões \\
\cline { 2 - 3 } Carbonatação & 1 & Localizada, com algumas regiões com pH<9, sem atingir a armadura \\
\cline { 2 - 3 } & 2 & Localizada, atingindo a armadura, em ambiente seco; \\
\cline { 2 - 3 } & 3 & Localizada, atingindo a armadura, em ambiente úmido \\
\cline { 2 - 3 } $\begin{array}{c}\text { Cobrimento } \\
\text { Deficiente }\end{array}$ & 4 & Generalizada, atingindo a armadura, em ambiente úmido \\
\cline { 2 - 3 } & 1 & Senores do que previsto em norma sem, no entanto, permitir a localização \\
da armadura
\end{tabular}




\section{\begin{tabular}{r|r|r}
\hline & 4 & Em ambientes úmidos \\
\hline
\end{tabular}}

Fonte: Euqueres, 2011.

\subsubsection{Grau do dano (D)}

O Grau do Dano (D) é determinado pelas equações 1 e 2 a partir do Fator de ponderação $\left(F_{p}\right)$ e do Fator de intensidade $\left(\mathrm{F}_{\mathrm{i}}\right)$ :

$$
\begin{gathered}
\mathrm{D}=0,8 \mathrm{Fi} \text { Fp para } \mathrm{Fi} \leq 2,0 \\
\mathrm{D}=(12 \mathrm{Fi}-28) \text { para } \mathrm{Fi} \geq 3,0
\end{gathered}
$$

\subsubsection{Grau de deterioração de um elemento $\left(G_{d e}\right)$}

O grau de Deterioração de um Elemento (Gde) é calculado em função do Grau do Dano (D), este parâmetro indica o nível de degradação de cada elemento individualmente (MEDEIROS, 2015). Conforme equação 3:

$$
\text { Gde }=\text { Dmáx }\left[1+\frac{\sum_{1=}^{\mathrm{m}} \mathrm{D}(\mathrm{i})-\mathrm{D}(\mathrm{máx})}{\sum_{\mathrm{i}=1}^{\mathrm{m}} \mathrm{D}(\mathrm{i})}\right]
$$

Onde:

$\mathrm{G}_{\mathrm{de}}=$ Grau de deterioração de um elemento;

$\mathrm{D}_{(\mathrm{i})}=$ é o grau de dano de ordem $\mathrm{i}$;

$\mathrm{D}_{\text {máx }}=$ maior dano detectado no elemento;

$\mathrm{m}=$ é o número de danos encontrados em um elemento

Em função do $\mathrm{G}_{\mathrm{de}}$ utiliza-se a Tabela 2, para a classificação dos níveis de deterioração no elemento, com recomendações

\begin{tabular}{|c|c|c|}
\hline $\begin{array}{c}\text { Nível de } \\
\text { deterioração }\end{array}$ & $\mathrm{G}_{\mathrm{de}}$ & Ações a serem adotadas \\
\hline Baixo & $\leq 15$ & Estado aceitável. Manutenção preventiva. \\
\hline Médio & 16 a 50 & $\begin{array}{l}\text { Definir prazo/natureza para nova inspeção. Planejar } \\
\text { intervenção em longo prazo (máx. } 2 \text { anos). }\end{array}$ \\
\hline Alto & 51 a 80 & $\begin{array}{l}\text { Definir prazo/natureza para inspeção especializada } \\
\text { detalhada. Planejar intervenção em médio prazo (máx. } \\
18 \text { meses). }\end{array}$ \\
\hline Sofrível & 81 a 100 & $\begin{array}{c}\text { Definir prazo/natureza para inspeção especializada } \\
\text { detalhada. Planejar intervenção em médio prazo (máx. } 1 \\
\text { ano). }\end{array}$ \\
\hline Crítico & $\geq 101$ & $\begin{array}{c}\text { Inspeção especial emergencial. Planejar intervenção } \\
\text { imediata. }\end{array}$ \\
\hline
\end{tabular}
das medidas a serem adotadas.

Tabela 2 - Classificação dos níveis de deterioração do elemento.

Fonte: Euqueres, 2011.

\subsubsection{Grau de deterioração de uma família de elementos $\left(\mathbf{G}_{\mathrm{df}}\right)$}

O Grau de Deterioração de uma Família de Elementos $\left(\mathrm{G}_{\mathrm{df}}\right)$ é definido como sendo a média aritmética dos Graus de Deterioração dos Elementos, conforme Equação 4, e representa a deterioração de um conjunto de elementos com a mesma característica.

$$
\mathrm{Gdf}=\text { Gdemáx }\left[\sqrt{1+\frac{\sum_{\mathrm{i}=1}^{\mathrm{m}} \mathrm{G}_{\mathrm{de}(\mathrm{i})}-\mathrm{G}_{\mathrm{demáx}}}{\sum_{\mathrm{i}=1}^{\mathrm{m}} \mathrm{G}_{\mathrm{de}(\mathrm{i})}}}\right]
$$

Onde: 


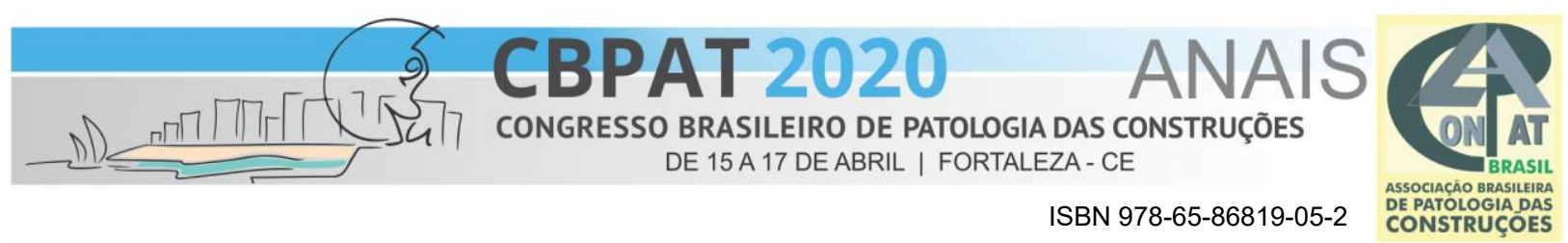

Gdf = Grau de deterioração de uma família; Gmáx = maior valor de Gde de uma família; $\mathrm{m}=$ número de elementos.

\subsubsection{Fator de relevância estrutural $\left(F_{r}\right)$}

Esse fator considera a importância de cada tipo de elemento, dentro do conjunto de elementos em que a obra é subdividida, em função de sua responsabilidade no comportamento estrutural e bom desempenho da mesma. Sua escala varia crescentemente com a importância do elemento, como se segue:

- Barreira de defesas e/ou Guarda-rodas

$$
\begin{aligned}
& \mathrm{Fr}=1,0 \\
& \mathrm{Fr}=2,0 \\
& \mathrm{Fr}=3,0 \\
& \mathrm{Fr}=4,0 \\
& \mathrm{Fr}=5,0
\end{aligned}
$$

- Pista de rolagem

- Cortinas, Juntas de dilatação

- $\quad$ Lajes, Fundações, Aparelhos de apoio, Vigas secundárias

- Vigas principais e Pilares

O item Fundações é classificado como item de segunda ordem, pois a metodologia contempla apenas inspeções visuais, não sendo possível a observância de todos os elementos de fundações em sua totalidade, desta forma esse item não mascara os resultados obtidos.

\subsubsection{Grau de deterioração da Estrutura $($ Gd)}

O Grau de Deterioração da Estrutura (Gd) é calculado através da média ponderada dos Graus de Deterioração das Famílias de Elementos e utiliza como pesos os Fatores de relevância estrutural (Fr), conforme Equação 5.

$$
\mathrm{Gd}=\frac{\sum_{\mathrm{i}=1}^{\mathrm{k}} \mathrm{F}_{\mathrm{r}(\mathrm{i})} \mathrm{G}_{\mathrm{df}(\mathrm{i})}}{\sum \mathrm{F}_{\mathrm{r}(\mathrm{i})}}
$$

Onde:

Fr - Fator de relevância estrutural de uma família de elementos;

Gdf - grau de deterioração da família.

Obs.: Para a classificação do nível de deterioração utilizamos a Tabela 1.

\section{RESULTADOS}

\subsection{Viaduto Miguel Arraes}

Inaugurado em 2008, este viaduto interliga as avenidas Mário Ypiranga, Ephigênio Salles e Av. Maceió. A análise dos elementos se deu com preenchimento in loco de tabelas. Após o preenchimento de todos os elementos, deu-se sequência aos cálculos conforme equação 1, 2 e 3. Os resultados de D, de Gde e Gdf podem ser observados na Tabela 3.

Tabela 3 - Resultados obtidos para o maior somatório do Grau de dano (D), Grau de Deterioração do Elemento (Gde) e Grau de Deterioração da Família de Elementos (Gdf) do viaduto Miguel Arraes

\begin{tabular}{c|c|c|c}
\hline Elemento & Maior $\sum$ Dano & $\mathrm{G}_{\mathrm{demax}}$ & $\mathrm{G}_{\mathrm{df}}$ \\
\hline Pilar 2 & 12,00 & 7,68 & 10,61 \\
\hline Viga 3 & 12,00 & 7,68 & 10,56 \\
\hline Laje 1 & 6,40 & 5,50 & 7,56 \\
\hline Barreira de defesa 6 & 47,20 & 35,80 & 48,12 \\
\hline Cortina 4 & 176,00 & 99,55 & 134,63 \\
\hline Pista 1 & 8,00 & 4,00 & 7,75 \\
\hline Junta de dilatação 4 & 48,00 & 46,67 & 80,22 \\
\hline
\end{tabular}


Com os resultados obtidos, observa-se que a Família de Cortinas é a que apresenta o maior Grau de Deterioração em nível Crítico com Gdf de 134,63, isso significa que os elementos dessa família necessitam de uma inspeção especial emergencial.

As famílias de Pilares, Vigas principais, Lajes e Pista possuem Grau de deterioração Baixo, que indica um estado estável, demandam apenas uma manutenção preventiva (a cada 5 anos). As Barreiras de defesa classificam-se em nível Médio, assim é necessário definir um prazo para nova inspeção e o planejamento de uma intervenção a médio prazo (máximo de 36 meses).

A família Junta de dilatação apresenta Grau de nível Alto $\left(\mathrm{G}_{\mathrm{df}}=80,22\right)$, assim de acordo com a Tabela 2 deve-se definir um prazo para nova inspeção sendo de natureza especializada e detalhada, e planejar uma intervenção com prazo máximo de 18 meses.

Para o Grau de Deterioração da Estrutura (Gd) calculou-se utilizando a Equação 5 incluindo os Fatores de relevância estrutural (Fr). Os resultados obtidos são apresentados na Tabela 4:

Tabela 4 - Resultados obtidos para o Grau de Deterioração da Estrutura (Gd), para o viaduto Miguel Arraes.

\begin{tabular}{c|c|c|c}
\hline Família & Gdf & Fr & Gdf x Fr \\
\hline Pilar & 10,61 & 5 & 53,06 \\
\hline Viga principais & 10,56 & 5 & 52,82 \\
\hline Laje & 7,56 & 4 & 30,24 \\
\hline Cortinas & 134,63 & 3 & 403,88 \\
\hline Junta de dilatação & 80,22 & 3 & 240,67 \\
\hline Pista & 7,75 & 2 & 15,49 \\
\hline Barreira de defesa & 48,12 & 1 & 48,12 \\
\hline \multicolumn{2}{c}{ Somatório } & 23 & 844,27 \\
\cline { 2 - 4 } & & Gd & 36,70 \\
\cline { 2 - 4 }
\end{tabular}

Apesar de alguns de seus elementos apresentarem nível de Deterioração Crítico, de acordo com a Tabela 2, o Grau de Deterioração da Estrutura do viaduto Miguel Arraes se classifica em nível Médio, com Gd= 36,70, isso se deve ao fato de famílias que apresentam maior Fator estrutural possuírem nível de deterioração Baixo, em relação aos demais elementos.

\subsection{Complexo viário Plínio Ramos Coelho}

Sua inauguração data de 1995, possuem dois viadutos independentes que interligam as Avenidas Torquato Tapajós e Mario Ypiranga, e Torquato Tapajós e Constantino Nery. A Figura 1 apresenta uma das famílias com maior deterioração encontrada no viaduto Plínio Ramos, que são as cortinas, com presença acentuada de manchas. A Tabela 5 e Tabela 6 apresenta os resultados das inspeções e as Tabelas 7 e 8 mostram os resultados do Grau de Deterioração da Estrutura para ambos os viadutos.

Figura 1 - Cortina do viaduto Plínio Ramos com presença de manchas.

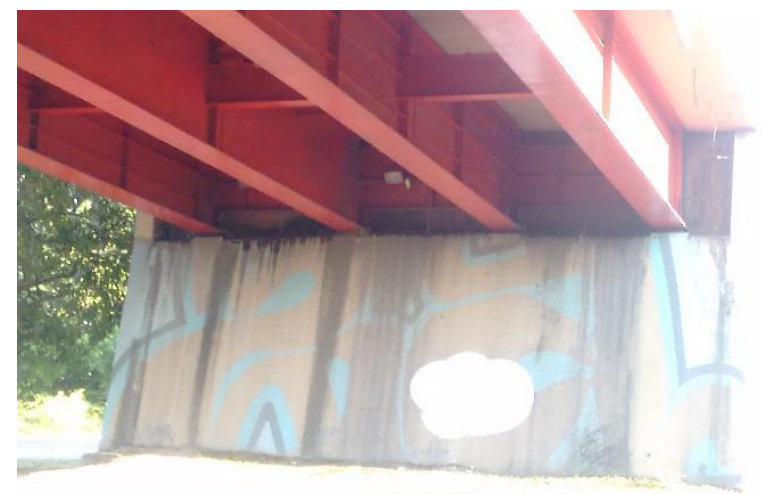


Tabela 5 - Resultados obtidos após análise do complexo viário Plínio Ramos Coelho, Av. Torquato Tapajós com Constantino Nery para o Maior somatório de Dano dos elementos, Grau de deterioração máximo (Gdemáx) e Grau de Deterioração da Família de Elementos (Gdf).

\begin{tabular}{c|c|c|c}
\hline Elemento & Maior $\sum$ Dano & Gdemax & Gdf \\
\hline Pilar 1 & 38,40 & 33,00 & 41,37 \\
\hline Viga 1 & 16,00 & 8,16 & 9,98 \\
\hline Laje & 8,08 & 8,08 & 8,08 \\
\hline Barreira de defesa 6 & 8,45 & 20,00 & 10,32 \\
\hline Cortina 2 & 31,20 & 29,54 & 38,18 \\
\hline Pista & 4,00 & 4,00 & 4,90 \\
\hline Junta de dilatação & \multicolumn{3}{|c}{ Não observado } \\
\hline
\end{tabular}

Tabela 6 Resultados obtidos após análise do complexo viário Plínio Ramos Coelho, Av. Torquato Tapajós com Av. Mário Ypiranga para o Maior somatório de Dano dos elementos, Grau de deterioração máximo (Gdemáx) e Grau de Deterioração da Família de Elementos (Gdf).

\begin{tabular}{c|c|c|c}
\hline Elemento & Maior $\sum$ Dano & Gdemax & Gdf \\
\hline Pilar 3 & 22,40 & 13,14 & 17,19 \\
\hline Viga 4 & 12,00 & 7,68 & 10,15 \\
\hline Laje 5 & 13,60 & 7,91 & 10,61 \\
\hline Barreira de defesa 2 & 15,20 & 8,08 & 9,90 \\
\hline Cortina 1 & 47,20 & 35,80 & 45,66 \\
\hline Pista & 12,00 & 6,67 & 6,67 \\
\hline Junta de dilatação 3 & 16,00 & 12,00 & 15,43 \\
\hline
\end{tabular}

Tabela 7 - Grau de Deterioração da Estrutura (Gd) Complexo viário Plínio Ramos Coelho: Av. Torquato Tapajós com Av. Constantino Nery.

\begin{tabular}{c|c|c|c}
\hline Família & Gdf & Fr & Gdf x Fr \\
\hline Pilar & 41,37 & 5 & 206,84 \\
\hline Viga principais & 9,98 & 5 & 49,89 \\
\hline Laje & 8,08 & 4 & 32,34 \\
\hline Cortinas & 38,18 & 3 & 114,55 \\
\hline Barreira de defesa & 10,32 & 1 & 10,32 \\
\hline Pista & 4,90 & 2 & 9,80 \\
\hline & Somatório & 20 & 423,73 \\
\cline { 2 - 4 } & \multicolumn{2}{|c}{ Gd } & 21,19 \\
\cline { 2 - 4 } & \multicolumn{2}{c}{}
\end{tabular}

Tabela 8 - Grau de Deterioração da Estrutura (Gd) Complexo viário Plínio Ramos Coelho: Av. Torquato Tapajós com Av. Mário Ypiranga.

\begin{tabular}{c|c|c|c}
\hline Família & Gdf & Fr & Gdf x Fr \\
\hline Pilar & 17,19 & 5 & 85,97 \\
\hline Viga principais & 10,15 & 5 & 50,77 \\
\hline Laje & 10,61 & 4 & 42,43 \\
\hline Cortinas & 45,66 & 3 & 136,99 \\
\hline Barreira de defesa & 9,90 & 1 & 9,90 \\
\hline
\end{tabular}




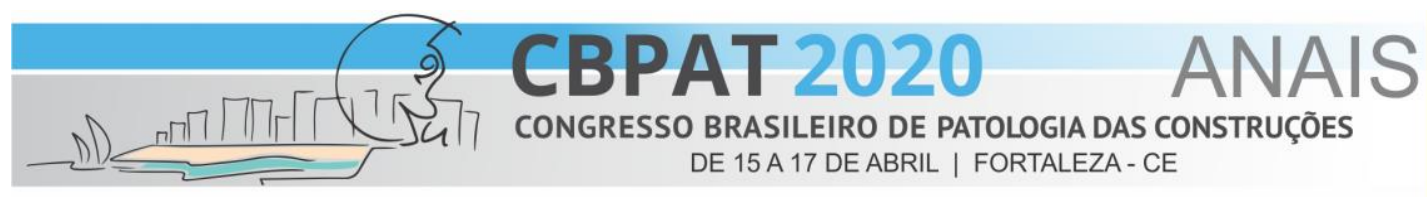

ISBN 978-65-86819-05-2

\begin{tabular}{|c|c|c|c|}
\hline Pista & 6,67 & 2 & 13,33 \\
\hline Junta de dilatação & 15,43 & 3 & 46,30 \\
\hline & Somatório & 23 & 385,69 \\
\hline & \multicolumn{2}{|c|}{ Gd } & 16,77 \\
\hline
\end{tabular}

No viaduto que liga a Av. Torquato Tapajós com a Av. Constantino Nery a família de Pilares apresenta o maior Grau de Deterioração $(\mathrm{Gdf}=41,37)$ e o viaduto que une as Avenidas Torquato Tapajós e Mario Ypiranga, a família de Cortinas apresentam maior Grau de Deterioração $(\mathrm{Gdf}=45,66)$ ambas as famílias se classificam em nível Médio. Ambos os viadutos se classificam em nível de deterioração Médio.

\subsection{Viaduto Ayrton Senna}

Este viaduto está localizado entre as Avenidas Djalma Batista e Mario Ypiranga inaugurado em 1995, na Tabela 9 constam os resultados para o Grau de deterioração máximo alcançado em cada família de elemento.

Tabela 9 - Resultados obtidos para o maior somatório do Grau de dano (D), Grau de Deterioração do Elemento (Gde) e Grau de Deterioração da Família de Elementos (Gdf) do viaduto Ayrton Senna.

\begin{tabular}{c|c|c|c}
\hline Elemento & Maior $\sum$ Dano & Gdemax & Gdf \\
\hline Pilar 7 & 114,40 & 88,53 & 199,24 \\
\hline Viga 6 & 98,40 & 94,96 & 118,35 \\
\hline Laje 3 & 103,20 & 64,50 & 86,16 \\
\hline Barreira de defesa 2 & 130,40 & 92,39 & 113,16 \\
\hline Cortina 1 & 254,40 & 105,85 & 129,64 \\
\hline Pista 1 & 6,67 & 6,67 & 6,67 \\
\hline Junta de dilatação 3 & 104,00 & 103,85 & 127,19 \\
\hline Aparelho de Apoio 2 & 42,40 & 42,26 & 51,79 \\
\hline
\end{tabular}

As famílias de Pilares, Vigas, Barreira de defesa, Cortina, e Junta de dilatação apresentam Deterioração em nível Crítico ( $\mathrm{Gd}>101)$, desta forma nesses elementos exige-se uma inspeção especial em caráter emergencial, sendo ainda necessário uma intervenção imediata. Na Figura 2 é possível verificar algumas patologias como: manchas, umidade e presença de vegetação em uma das vigas do viaduto Ayrton Senna.

Figura 2 - Viga com danos de manchas, sinais de umidade e presença acentuada de vegetação.

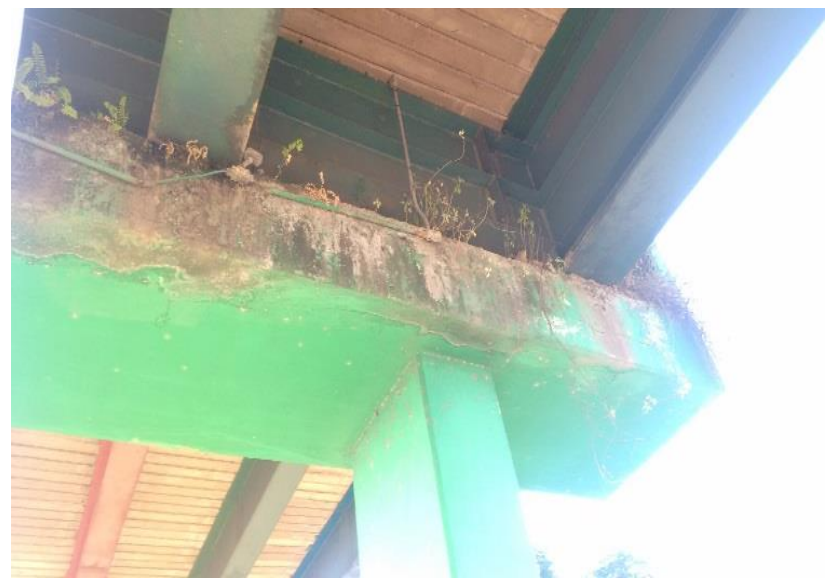

As lajes estão em um nível sofrível de deterioração $(\mathrm{Gdf}=86,16)$ sendo então exigido uma intervenção em um prazo máximo de 12 meses. Aparelho de apoio (Figura 3) apresenta deterioração de nível médio, com fissuras por esmagamento que ocorre basicamente por concentração de tensões, que podem ser provocadas por um carregamento adicional, aumentando assim os esforços de compressão no elemento estrutural, ou por falha em um dos componentes da estrutura, 


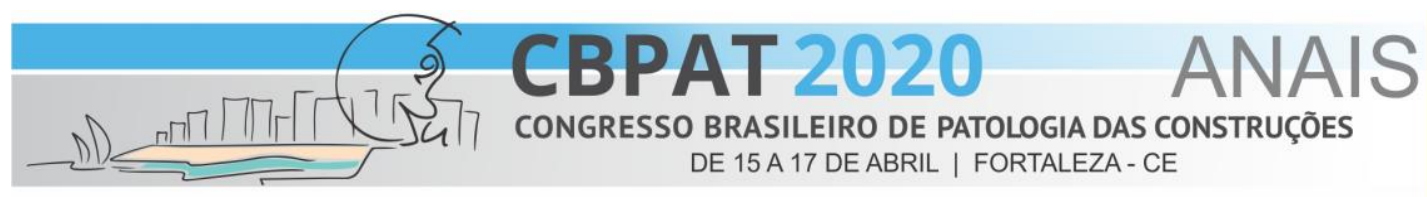

ISBN 978-65-86819-05-2

geralmente no aparelho de apoio (VERLY, 2015). Na Tabela 10 são apresentados os resultados obtidos a partir da análise global da estrutura:

Figura 3 - Aparelho de apoio com sinais de esmagamento.

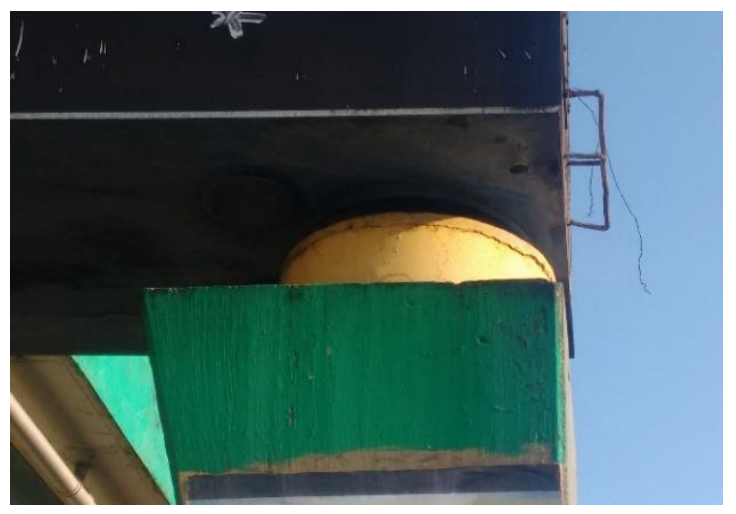

Tabela 10 - Grau de Deterioração da Estrutura (Gd) Viaduto Ayrton Senna.

\begin{tabular}{c|c|c|c}
\hline Família & Gdf & Fr & Gdf x Fr \\
\hline Pilar & 110,42 & 5 & 552,11 \\
\hline Viga principais & 118,35 & 5 & 591,73 \\
\hline Laje & 86,16 & 4 & 344,65 \\
\hline Aparelhos de apoio & 51,79 & 4 & 207,16 \\
\hline Cortinas & 129,64 & 3 & 388,91 \\
\hline Barreira de defesa & 113,16 & 1 & 113,16 \\
\hline Pista & 6,67 & 2 & 13,33 \\
\hline Junta de dilatação & 127,19 & 3 & 381,56 \\
\hline & Somatório & 27 & 2592,61 \\
\cline { 2 - 4 } & & Gd & 96,02 \\
\cline { 2 - 4 }
\end{tabular}

A análise da estrutura em aspecto global apresenta nível de deterioração Sofrível, com Gd=96,02, pode-se admitir que a melhor opção de intervenção neste caso é adotar uma ação em caráter emergencial e localizada, principalmente nas vigas e pilares, ação recomendada por Euqueres (2011).

\subsection{Viaduto Duca Brito}

O viaduto Duca Brito está localizado na Av. Djalma Batista com Av. Álvaro Maia, e foi inaugurado em 1998. Os resultados da sua inspeção encontram-se na Tabela 11, onde é descrito o elemento com maior somatório de danos, o Grau de Deterioração máximo dentro da Família $\left(\mathrm{G}_{\mathrm{demáx}}\right)$, e por fim o Grau de Deterioração da Família de elementos $\left(\mathrm{G}_{\mathrm{df}}\right)$.

Tabela 11 - Resultados obtidos para o maior somatório do Grau de dano (D), Grau de Deterioração do Elemento (Gde) e Grau de Deterioração da Família de Elementos (Gdf) do viaduto Duca Brito.

\begin{tabular}{c|c|c|c}
\hline Elemento & Maior $\sum$ Dano & Gdemax & Gdf \\
\hline Pilar & 28,80 & 4,60 & 4,60 \\
\hline Laje 1 & 32,80 & 30,44 & 37,28 \\
\hline Barreira de defesa 3 & 96,90 & 82,81 & 111,07 \\
\hline Cortina 3 & 112,80 & 88,09 & 115,52 \\
\hline Pista 1 & 8,00 & 6,00 & 7,98 \\
\hline
\end{tabular}




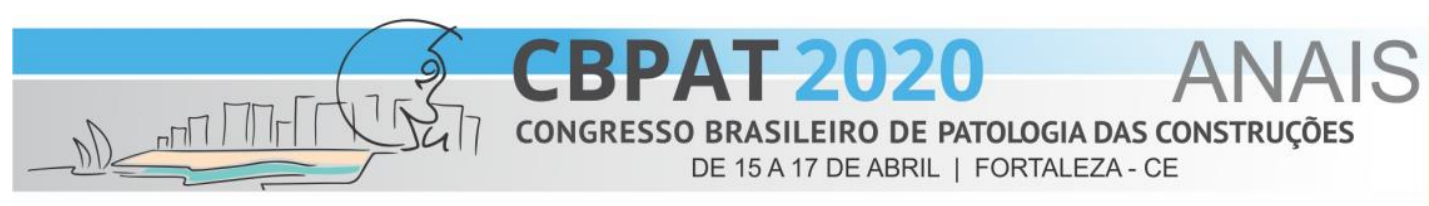

ISBN 978-65-86819-05-2

As famílias de Barreira de defesa e de Cortina são as que apresentam maior nível de deterioração sendo classificadas com nível Sofrível, para este nível é necessário planejar uma inspeção emergencial e juntamente com uma intervenção imediata. A seguir foi calculado o nível de deterioração global da estrutural, os resultados podem ser analisados na Tabela 12:

Tabela 12 - Grau de Deterioração da Estrutura (Gd) Viaduto Duca Brito.

\begin{tabular}{c|c|c|c}
\hline Família & Gdf & Fr & Gdf x Fr \\
\hline Pilar & 4,60 & 5 & 23,00 \\
\hline Laje & 37,28 & 4 & 149,12 \\
\hline Cortinas & 115,52 & 3 & 346,56 \\
\hline Barreira de defesa & 111,07 & 1 & 111,07 \\
\hline Pista & 7,98 & 2 & 15,96 \\
\hline & Somatório & 15 & 645,71 \\
\cline { 2 - 4 } & \multicolumn{2}{|c|}{ Gd } & 43,05 \\
\cline { 2 - 4 }
\end{tabular}

Resultados semelhantes foram encontrados no estudo de Silva e Moura (2017) aplicados em viadutos em Brasília, o nível de deterioração alcançado para estrutura Médio, apesar de apresentar elementos em nível Sofrível, isto ocorre porque o fator de relevância estrutural para a família de Barreiras e Cortinas é baixo em termos estruturais, mas a curto prazo deve haver intervenção nesses elementos.

\subsection{Dom Jackson Damasceno Rodrigues}

Inaugurado em 1998, localiza-se na interseção das Avenidas Constantino e Boulevard Álvaro Maia. Na Figura 4, é possível observar o pilar com maior somatório de dano, apresentando fissuras e desplacamento. Na Tabela 13 são expostos os resultados da aplicação da Metodologia Gde/UnB e na Tabela 14 temos o resultado da análise global da estrutura.

Figura 4 - Base do pilar do viaduto Dom Jackson Damasceno com sinais de esmagamento, armadura exposta com indícios de corrosão.

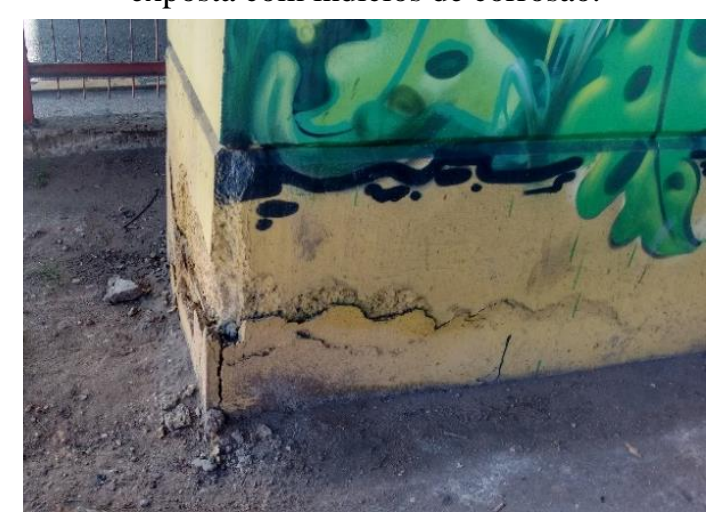

Tabela 13 - Resultados obtidos para o maior somatório do Grau de dano (D), Grau de Deterioração do Elemento (Gde) e Grau de Deterioração da Família de Elementos (Gdf) do viaduto Dom Jackson Damasceno Rodrigues.

\begin{tabular}{c|c|c|c}
\hline Elemento & Maior $\sum$ Dano & Gdemax & Gdf \\
\hline Pilar 3 & 98,40 & 63,74 & 83,02 \\
\hline Viga 6 & 15,20 & 8,08 & 10,93 \\
\hline Laje 6 & 5,60 & 5,14 & 6,96 \\
\hline Barreira de defesa 1 & 48,80 & 47,21 & 49,87 \\
\hline Cortina 2 & 76,80 & 73,13 & 78,91 \\
\hline Pista 1 & 8,00 & 6,00 & 6,00 \\
\hline Junta de dilatação 3 & 8,00 & 6,00 & 8,05 \\
\hline
\end{tabular}




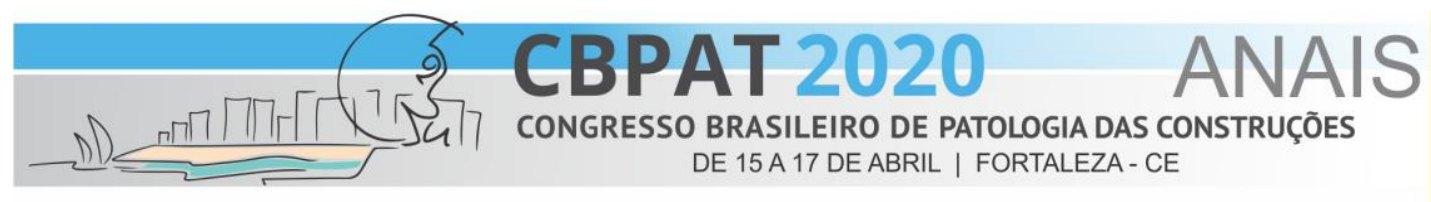

ISBN 978-65-86819-05-2

Aparelho de Apoio 2

68,00

63,75

86,35

Tabela 14 - Grau de Deterioração da Estrutura (Gd) Viaduto Dom Jackson Damasceno.

\begin{tabular}{|c|c|c|c|}
\hline Família & Gdf & $\mathrm{Fr}$ & Gdf $x$ Fr \\
\hline Pilar & 83,02 & 5 & 415,08 \\
\hline Viga principais & 10,93 & 5 & 54,63 \\
\hline Laje & 6,96 & 4 & 27,85 \\
\hline Aparelhos de apoio & 86,35 & 4 & 345,41 \\
\hline Cortinas & 78,91 & 3 & 236,73 \\
\hline Barreira de defesa & 49,87 & 1 & 49,87 \\
\hline Pista & 7,35 & 2 & 14,70 \\
\hline Junta de dilatação & 8,05 & 3 & 24,15 \\
\hline & Somatório & 27 & 1168,43 \\
\hline & \multicolumn{2}{|c|}{ Gd } & 43,28 \\
\hline
\end{tabular}

As famílias de elementos que apresentam maior nível de deterioração são as famílias de Pilares e Aparelho de apoio, com Gdf classificado em nível Alto. A análise global, indica, porém, uma deterioração em nível Médio, este fato se dá, pois, as outras famílias de elementos tiveram grau de deterioração baixo, fazendo com que a estrutura seja classificada em nível de deterioração Médio, Euqueres (2011) em seu estudo com pontes em Goiânia alcança resultados semelhantes e indica o prazo máximo de ações de 36 meses para uma nova inspeção

\section{CONCLUSÕES}

Para realizar as análises dos diversos viadutos a principal dificuldade foi o acesso as partes superiores dos viadutos, impossibilitando em certos casos a visualização de todos os elementos, assim como também afirma Verly (2015) e Euqueres (2015) em seus trabalhos. Ao comparar os valores de Gd com o estado atual dos viadutos confirma-se que a metodologia é eficiente para a aplicação in loco por meio de análises visuais, pois retrata a realidade.

A aplicação da metodologia GDE/UnB possibilitou identificar diversas manifestações patológicas como fissuras, manchas, presença de vegetação e umidade, exposição de armaduras e áreas com concreto desplacando. Os viadutos Miguel Arraes, Duca Brito e Dom Jackson Damasceno e o complexo viário Plínio Ramos encontram-se em nível médio de deterioração, deve-se planejar uma intervenção de longo prazo (máx. 2 anos) em toda estrutura desses viadutos.

Alguns dos elementos dos viadutos citados apresentam grau de deterioração alto, como Cortinas e Barreira de defesa no viaduto Duca Brita, Aparelhos de apoio e pilares no Dom Jackson Damasceno, nestes casos deve-se planejar uma intervenção em médio prazo, para inspeção especializada e detalhada, com prazo máximo de 18 meses dessas famílias. Em relação ao viaduto Miguel Arraes que possui Família de Elemento em nível crítico, como é o caso da família de cortinas, a metodologia indica uma intervenção imediata nessa família.

O viaduto Ayrton Senna classifica-se em nível sofrível de deterioração, para este o prazo de intervenção é de no máximo 1 ano. Para as Famílias de Pilares, Vigas, Barreira de defesa, Cortina, e Junta de dilatação que se encontram em nível crítico de deterioração, recomenda-se planejar uma intervenção imediata de caráter emergencial.

A maior incidência de danos encontrados foram fissuras e manchas, resultados semelhantes aos das inspeções de Medeiros (2015) e Verly (2015). As fissuras se apresentaram em maior quantidade nas vigas e pilares, enquanto as manchas encontram-se principalmente nas cortinas e vigas. Vale ressaltar ainda, que mesmo nas estruturas onde tiveram como resultado Grau de Deterioração médio há itens com nível de deterioração mais elevados, isso norteia as ações para serem adotas em cada estrutura, de acordo com sua família.

As principais patologias identificadas se desenvolveram pela falta de manutenção periódica, uma possível solução para esse problema é a implantação de um sistema de gerenciamento de obras de artes especiais, que possibilita manter um cadastro atualizado e de planejamento para ações de correção nessas estruturas.

\section{REFERÊNCIAS}

AlbuQuerQue, A. T de; OTOCH, S. Proposta de classificação da agressividade do ambiente na cidade de Fortaleza. Anais do $47^{\circ}$ Congresso Brasileiro do Concreto. Recife, 2005. 


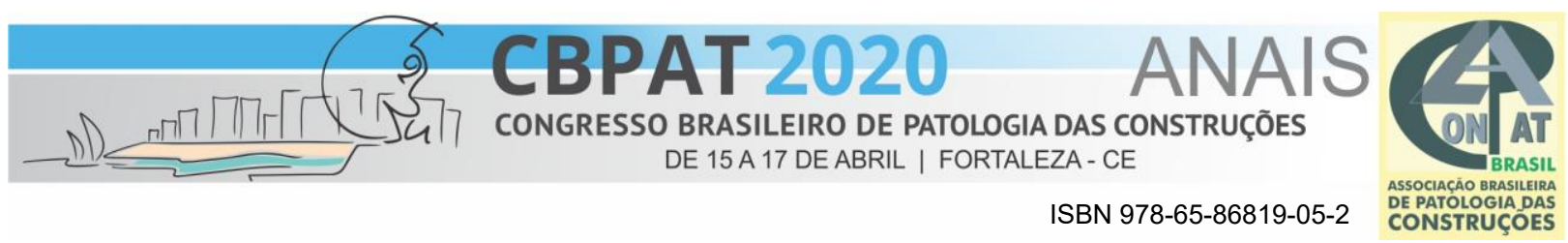

BARBOSA, G. A; BASTOS. C; BARRETO, M. F. Estudo das manifestações patológicas em pontes e viadutos de concreto armado na cidade de Cuiabá - MT. Anais do $2^{\circ}$ Encontro Latino-Americano e Europeu sobre Edificações e comunidades sustentáveis. São Leopoldo, 2017.

BASTOS, H. C. N; MIRANDA, M. Z. Principais patologias em estruturas de concreto de pontes e viadutos: Manuseio e manutenção das obras de arte especiais. Construindo, Belo Horizonte, v. 9, p. 93-101, jul./dez., 2017.

CASTRO, E. K. Desenvolvimento de Metodologia de Estruturas de Concreto Armado. 1994. 129 p. Dissertação (Mestrado). Faculdade de tecnologia. Universidade de Brasília, 1994.

EUQUERES, PRISCILA. Metodologia de Inspeção em Estruturas de Pontes de Concreto Armado. 2011.170 p. Dissertação (Mestrado). Escola de Engenharia Civil. Universidade Federal de Goiás, 2011.

FONSECA, R. P. A estrutura do Instituto Central de Ciências: Aspectos históricos, científicos e tecnológicos de projeto, execução, intervenções e propostas de manutenção. 2007. 213p. Dissertação (Mestrado em Estruturas e Construção Civil), Universidade de Brasília. Brasília, 2015.

FONTE, F. J. M; VITÓRIO, J. A. P. Proposta para um sistema de inspeção e manutenção dos túneis da cidade do Recife. In: Anais do $7^{\circ}$ Congresso Internacional sobre Patologia e Reabilitação de Estruturas. Fortaleza, 2011.

FRANÇA, R.M. M; MONTEIRO, E. C.B. Análise das Manifestações Patológicas Encontradas em Edificação Residencial Unifamiliar no Agreste Pernambucano. Revista e Pesquisa Aplicada. Pernambuco, vol 1. p. 29--33

MEDEIROS, A. G. Analise de durabilidade da Ponte do Rio do Carmo utilizando ensaios não destrutivos, Norma DNIT e a Metodologia GDE/UnB. 165 p. Dissertação (Mestrado em Engenharia Civil). Universidade Federal do Rio Grande do Norte. Natal, 2015.

MEDEIROS, M. H. F. de; ANDRADE, J. J. O; HELENE, P. Vida Útil das Estruturas de Concreto. In: ISAIA, G. C. Concreto ciência e Tecnologia. São Paulo: IBRACON, 2011 vol. 2.

MEHTA, P. K; MONTEIRO P. J. M. Concreto: Microestrutura, propriedades e materiais. 2 ed. IBRACON, 2014.

REUNION INTERNATIONALE DE LABORATOIRES D'ESSAIS ET MATERIAUX. CPC-18: Measurement of hardened concrete carbonation depth. RILEM Recommendations CPC-18. Materials and Structures, 1988.

SILVA, A. J. M; MOURA, M. P. E. Análise de manifestações patológicas em viadutos do DF. 2017. 34p. Trabalho de conclusão de curso (Bacharel). Universidade Católica de Brasília, 2017.

VERLY, R. C. Avaliação de metodologias de inspeção como instrumento de priorização de intervenções em obras de arte especiais. 2015. 178p. Dissertação (Mestrado em Estruturas e Construção Civil), Departamento de Engenharia Civil e Ambiental, Universidade de Brasília. Brasília, 2015.

VITÓRIO, J. A. P. Pontes rodoviárias: Fundamentos, conservação e gestão. Recife: CREA - PE, 2002. 